\title{
Latest Developments in Surgical and Minimally Invasive Treatment of Metastatic Bone Disease
}

\author{
Brian J. Kistler · Timothy A. Damron
}

Published online: 1 March 2014

(C) Springer Science + Business Media New York 2014

\begin{abstract}
Surgical treatment of metastatic disease to bone continues to evolve. Advances have been made in diagnosis, improved surgical outcomes, and minimally invasive procedures. Improved prediction of risk for bone fracture continues to evolve with CT-based techniques including structural rigidity analysis and finite element modeling. Improved surgical outcomes have been seen in treatment of proximal femoral pathologic lesions and fractures with the pendulum swinging toward more use of endoprosthetic devices over internal fixation, humeral lesions with a wide variety of techniques, and acetabular lesions with the use of tantalum acetabular cups and augments. Minimally invasive techniques including osteoplasty, radiofrequency ablation, cryoablation, and high-intensity ultrasound offer alternatives to open surgical treatment. Despite this evolution, the goals of treatment remain the same: pain relief, immediate stability, local disease control, and maintenance or restoration of function.
\end{abstract}

Keywords Metastatic bone disease - Pathologic fracture · Structural analysis - Augmented acetabular reconstruction . Radiofrequency ablation · Cryoablation · High-intensity focused ultrasound

This article is part of the Topical Collection on Ortho-oncology.

\section{B. J. Kistler}

SUNY Upstate Medical University, 750 East Adams St, Syracuse, NY 13210, USA

e-mail: Wiggin80@gmail.com

\section{T. A. Damron $(\bowtie)$}

Department of Orthopedics, SUNY Upstate Medical University, Suite 200, Upstate Bone and Joint Center, 6620 Fly Road, East Syracuse, NY 13057, USA

e-mail: DamronT@upstate.edu

\section{Introduction}

Over 10 million Americans are currently living with cancer, with the incidence in the USA estimated at 1.4 million annually [1]. Patients are living longer because of advancements in medical treatments. However, this also places these patients at an increased risk of developing metastatic bone disease [2]. The exact incidence of metastatic disease to the bone is unknown, but it is estimated that 350,000 people die with bony metastases each year in the USA [3].

Metastatic disease can affect various locations in the human body with the skeleton being the third most common behind metastatic disease to the lungs and liver [3, 4]. Common sources of metastases to the bone include the lung, kidney, thyroid, breast, and prostate, which together account for $80 \%$ of all skeletal metastases. In addition, 60-84\% of all cases of metastatic cancer involve metastases to the bone, and, of those, $70 \%$ of patients experience pain associated with their bone metastases [5].

Metastatic bone disease, although not the primary site of cancer, can be the source of significant morbidity. Pain secondary to metastatic bone disease can cause loss of function and decreased quality of life. In addition to pain, structural insufficiency with actual or impending pathologic fractures can result and significantly affect a patient's ability to perform simple tasks and activities of daily living [6].

Treatments for metastatic bone disease are usually not curative but rather are primarily palliative. In general, treatment does not prolong a patient's life expectancy, but it should be planned to improve the quality of the patient's remaining life. The specific goals of treatment are to relieve pain, gain local tumor control, and maintain or regain functional status. Long-term survival of patients after their first long bone pathologic fracture from 
malignancy has more than tripled for the most common cancers during the past 25 years [2]. With increased life expectancy after diagnosis and treatment, achieving the treatment goals and maintaining durability of the reconstruction become even more important.

Like so many other areas of medicine, the treatment of metastatic bone disease has continued to advance. Formal surgical treatments and minimally invasive procedures have evolved with the development of new technology. In addition to advances in treatment, there have also been new techniques in diagnosis and tools to help the physician more accurately predict which cases of metastatic bone disease require operative management and which can be treated with other treatments or even observed. Minimally invasive procedures are now becoming more common and showing promising results for the safe and effective treatment of metastatic bone disease. The purpose of this article is to review the latest developments in surgical and minimally invasive treatment of metastatic bone disease. The focus is on involvement in the long bones and acetabulum, as treatment of metastatic spinal disease, which has also undergone significant advances, is beyond the scope of this article.

\section{Impending Pathologic Fracture Prediction}

The presence of a pathologic fracture in a weight-bearing long bone has long been an accepted indication for surgical management. The goals of treatment are to alleviate pain and restore function by allowing immediate weight bearing in the lower extremity and functional use of the upper extremity. Likewise, prophylactic treatment by internal fixation of impending pathologic fractures has also been an accepted indication for surgical management in patients with metastatic bone disease, myeloma, and lymphoma. Ward et al. [7] showed better outcomes in patients who were treated with prophylactic treatment for impending pathologic fractures compared to patients who were treated after the injury for completed pathologic fractures. Advantages included decreased blood loss, shorter hospital stays, greater likelihood of discharge to home, and increased chance of resuming support-free ambulation.

The challenge to surgeons and clinicians has always been to determine which metastatic bone lesions are actually going to lead to pathologic fractures and for which prophylactic stabilization of the lesions would be appropriate versus those that may be safely observed and/or treated nonoperatively. Mirels [8], in his now classic article, proposed a scoring system to quantify the risk of sustaining a pathologic fracture through a known metastatic bone lesion.

Mirels' rating system was designed to predict the probability of fracture based on four parameters: the site of the lesion, pain from the lesion, nature of the lesion, and
Table 1 Mirels classification

\begin{tabular}{llll}
\hline & 1 & 2 & 3 \\
\hline Location & $\begin{array}{l}\text { Upper } \\
\text { extremity }\end{array}$ & $\begin{array}{c}\text { Other lower } \\
\text { extremity }\end{array}$ & Peritrochanteric \\
Nature & Blastic & Mixed & Lytic \\
Pain & Mild & Moderate & Severe \\
Size $^{\mathrm{b}}$ & $<1 / 3$ & $1 / 3-2 / 3$ & $>2 / 3$ \\
\hline
\end{tabular}

Mirels' Metastatic Disease in Long Bones. A proposed scoring system for diagnosing impending pathologic fractures

Adapted from Mirels [8]

a Other lower extremity refers to lower extremity sites other than the peritrochanteric region

b Size of the lesion relative to the width of the bone

size of the lesion (Table 1). Based on his rating system, a score of eight corresponded with a fracture probability of $15 \%$, a score of nine corresponded with a fracture probability of $33 \%$, and a score of ten corresponded with a fracture probability of $72 \%$. Nonoperative care was recommended for a score of 7 or less, the treating physician was recommended to consider stabilization for a score of 8 , and operative prophylactic stabilization was recommended for a score of 9 or above [8, 9].

Several studies have gone on to investigate the reproducibility and validity of Mirels' rating system [9-11]. These studies have shown that Mirels' scoring system is in fact reproducible and valid; however, the use of Mirels' rating system has been criticized for poor specificity [9]. In one review utilizing Mirels' rating system for a series of 12 patients, the true clinical histories and radiographs were reviewed by 53 participants, at five experience levels, and showed the overall sensitivity of a Mirels' score of 9 or above as $91 \%$, but the specificity was only $35 \%$ [9]. El-Husseiny et al. [12] found similar results when comparing inter- and intraobserver reproducibility, but found that a conventional system by Carnesale showed better inter- and intraobserver reliability. In one prospective study of patients undergoing radiotherapy, Mirels' scoring system was not found to be sufficiently specific (36\%) to predict fracture [13]. Although most of the work on Mirels' evaluation has focused on the femur, in evaluating humeral lesions Evans et al. [11] found that Mirels' score of 9 and above had a sensitivity and specificity of 14.5 and $82.9 \%$, respectively. In order to achieve a sensitivity and specificity similar to that of Mirels' cutoff of 9 points in their original paper focusing on the femur, other potential definitions of impending humeral pathologic fractures were evaluated. A cutoff of 7 points for those humeral lesions yielded a sensitivity and specificity of 81 and $32 \%$, respectively [11].

Over recent years, physicians and surgeons have begun using CT-based structural rigidity analysis (CTRA) to predict fracture risk $[14,15]$. This method is based on laboratory 
evaluations showing that the force required to fracture a bone with a simulated lytic lesion is related to the amount of reduction in structural rigidity of the weakest cross section through the bone $[14,15]$. A reduction of $35 \%$ or greater in axial, bending, or torsional rigidity compared to the intact contralateral side or to a matched bone (when the contralateral side is also involved or is not able to be evaluated because of prior fixation or replacement) has been used thus far as the definition of impending pathologic fracture. This model as well as fracture definition has been applied to a series of pediatric patients who presented with a fracture through a benign skeletal lesion compared to similar patients who had a benign skeletal lesion that had been thought to be at increased risk for fracture, had not fractured at the time of the study, underwent no surgical treatment, and had been followed for a minimum of 2 years [14]. The combination of bending and torsional rigidity measured noninvasively with quantitative CT was more accurate (97\%) for predicting pathologic fracture through benign bone lesions in children than were standard radiographic criteria (42-61\%). The same group then prospectively looked at 41 pediatric patients with benign skeletal lesions using the CTRA to predict fracture risk [15]. Of the 34 patients who completed followup criteria 2 years after initial CT-based analysis, none of the patients for whom no increased fracture risk had been predicted by the rigidity analysis sustained a fracture, even though they had not been treated surgically. This study method was more specific ( $97 \%$ specificity) than traditional radiographic methods [9]. While the use of CT-based examination in pediatric patients may be controversial because of the additional radiation exposure needed, the success of the technique has provided a basis for investigating its use in adult patients with metastatic disease, where the additional radiation exposure is of little consequence in these terminal patients.

CT-based structural analysis has been investigated in prospective studies in adult patients with skeletal bone lesions (Fig. 1). Although no clinical studies have been published to date, it is possible that CT-based structural analysis will prove as useful and reliable in the adult metastatic bone disease population as it has in the pediatric benign lesion population. Finite element modeling has also
Fig. 1 CT-based structural rigidity analysis (CTRA). In this femoral lesion with lytic characteristics, selected sagittal and axial CT slices are shown illustrating the region of maximal involvement by the lesion in question. The graph at the top was obtained from the CTRA report, showing the reductions in torsional, bending, and axial rigidity at the site of the defect. Note that while the reductions are notable compared to the intact adjacent regions of the femur, even the greatest drop (in axial rigidity) does not meet the threshold of a $35 \%$ reduction in rigidity that defines an impending pathologic fracture according to this technique. Hence, by CTRA, this patient's femur was deemed NOT at increased risk of pathologic fracture. Based upon Mirels' classification, this patient was given 3 points each for size and nature, 2 points for location, and 2 points for pain, for a total of 10 points, qualifying as an impending pathologic fracture. Preliminary multi-institutional results show that CTRA is more specific than Mirels for fracture prediction
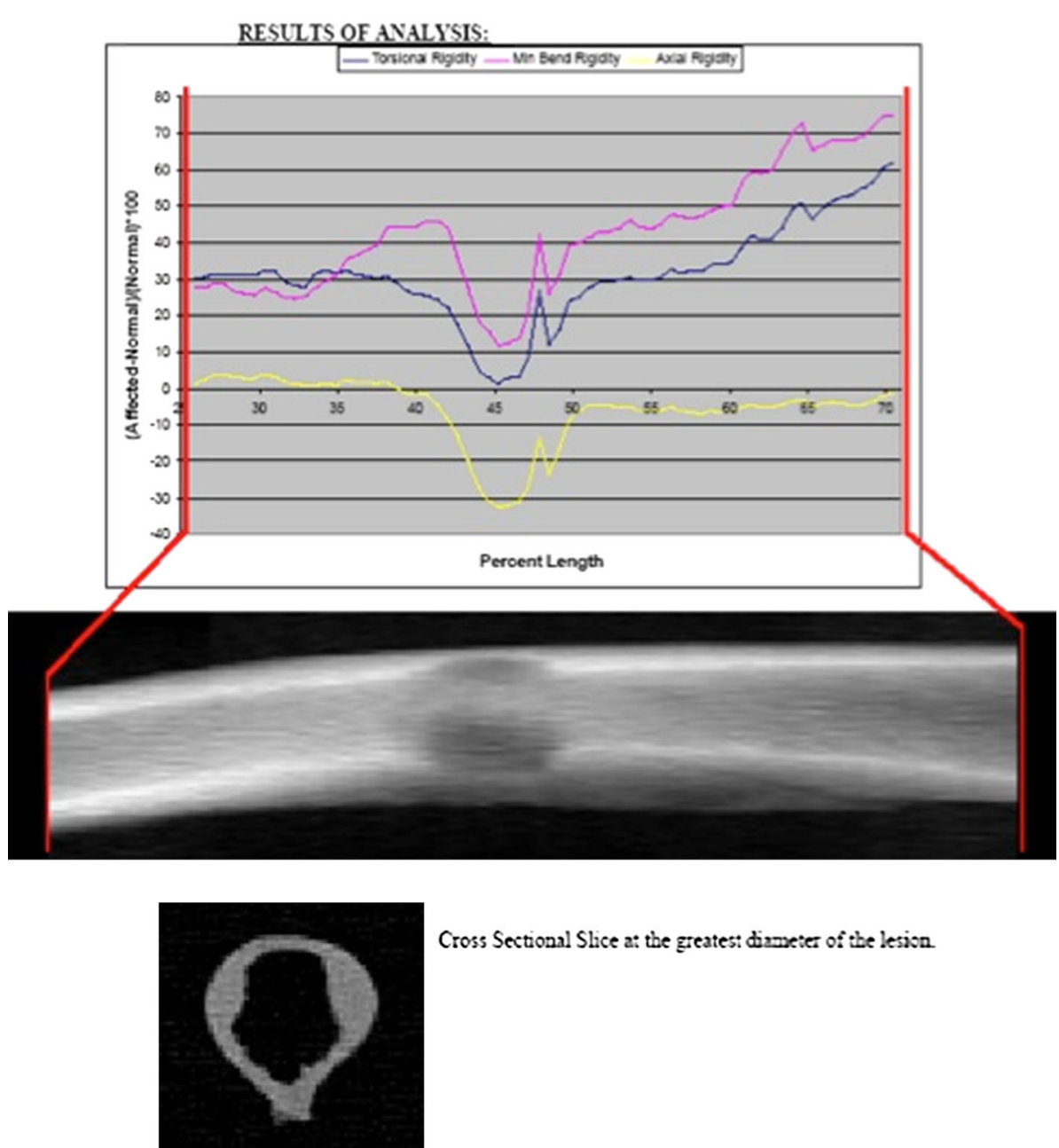

Cross Sectional Slice at the greates: dinareter of the lesion. 
Fig. 2 Standard treatment for impending or actual femoral neck fracture. This patient presented with right groin pain and a solitary lesion of the right femoral neck. Anteroposterior (a) and frog lateral (b) plain radiographs show the destructive femoral neck lesion. The CT scan (c) shows cortical disruption in the superior femoral neck without more distal lesions. Biopsy showed metastatic carcinoma, and the patient underwent long-stem cemented hemi-arthroplasty (d). She had excellent pain relief and was able to fully weight bear immediately after the operation. Since the CT scan of the entire femur did not show any evidence of other lesions, this patient may have been fine with a shorter stem, which would have decreased the risk of the pulmonary embolic phenomenon associated with longer cemented stems
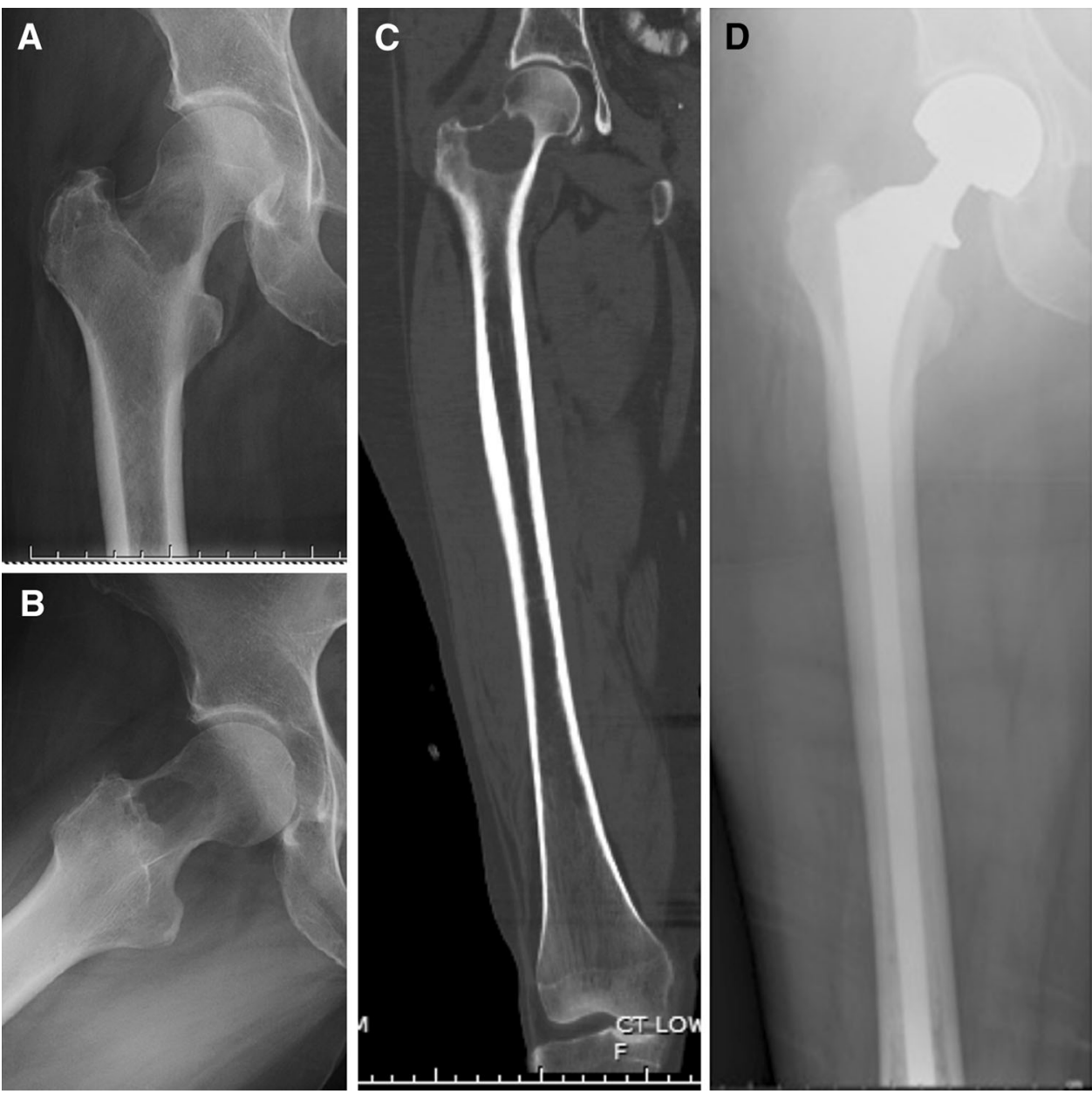

been evaluated in the laboratory as another means of impending fracture prediction, but its widespread application awaits clinical validation $[16,17]$.

\section{Surgical Treatments of Metastatic Bone Disease}

\section{Femoral Metastatic Disease}

The surgical care for impending and actual pathologic fractures of the femur has become fairly well established in most respects. For pathology of the femoral neck and head, particularly if a fracture has occurred, a hemiarthroplasty or total hip arthroplasty is generally recommended (Fig. 2). For more distal lesions, particularly when impending, a locked reconstruction femoral nail is usually employed. For more extensive proximal destruction, arthroplasty is favored over intramedullary nail fixation. For destruction limited to the level of the calcar at or above the lesser trochanter, calcar replacement longer stem implants are generally sufficient, but for destruction extending more distally, megaprosthesis reconstruction may be needed.
Cemented long-stem arthroplasty devices are still commonly used, although there has been interest in the use of shorter stems and non-cemented devices to minimize the significant embolic risk of the long-stem cemented devices [18-20].

The biggest controversy relative to surgical treatment of femoral metastatic disease relates to the operative treatment of intertrochanteric and subtrochanteric impending and pathologic fractures, which may involve either internal fixation or endoprosthetic reconstruction (Fig. 3). The advantage of internal fixation is the simplicity, availability, lower cost, and ease of application in a broader array of institutions. Further, internal fixation avoids the unique potential complications of arthroplasty, including instability and loosening, and the infection rate in most series is lower. The advantages of arthroplasty reconstruction include the immediate stability with less reliance on the integrity of the bone or need for healing. Hence, arthroplasty minimizes the complications of new or progressive fractures and loss of fixation inherent to internal fixation techniques. Recently, Sarahudi et al. [21] in a retrospective study reported comparative results for femoral metastatic cases, showing a 
higher failure rate of nearly $9 \%$ among 23 patients treated with internal fixation compared to $3.2 \%$ in 94 patients treated with arthroplasty. In a larger series by Steensma et al. [22], treatment failure was similarly lower among the arthroplasty patients $(3.1 \%)$ compared to internal fixation, but the most remarkable difference was between intramedullary nailing $(6.1 \%)$ and plate fixation $(42.1 \%)$. Not all of the arthroplasty complications required revision, while all of the failures of nail and plate fixation did, so the revision rate for arthroplasty of $0.5 \%$ was a clear advantage. In another recent large series, Harvey et al. [23•] showed comparable overall complication rates between intramedullary nailing $(26 \%)$ and arthroplasty (18\%), but a markedly lower rate of mechanical failure with the arthroplasties ( $0 \%$ compared to $11 \%$ ). Based on these series, the pendulum appears to have begun to swing toward arthroplasty for intertrochanteric and subtrochanteric metastatic disease. However, each case must be evaluated on its own merits, and the selection of either arthroplasty or internal fixation must be individualized to the patient, taking into consideration not only the anatomic location, extent of destruction, and underlying pathology, but also the expected duration of survival, patient expectations, and other comorbidities.

The role of locked plates in pathologic fracture and impending fracture fixation has yet to be defined [24]. While the poor results with plating in this context in the past were associated with earlier types of plate fixation, many of those are no longer in use. Unfortunately, there is there is a paucity of published data on biomechanical and clinical outcomes with the use of locking plates in the musculoskeletal oncology literature, and further clinical trials need to be published before we can truly see what the advantages and disadvantages of locking plates have in treating metastatic bone disease over conventional plates and possibly even intramedullary nails.

Over the past decade and a half, locked plating has become a popular mode of fixation in the osteosynthesis of traumatic fractures [25, 26]. Osteoporosis in the setting of trauma has become a common indication for the use of locked plating constructs $[26,27]$. The rationale for the use of locked plating systems is that loss of screw purchase becomes an ever more important factor related to failure of plate/screw fixation in osteoporotic bone. In traditional non-locked compression screws, the plate is secured to the bone as the screw is tightened. The friction created between the plate and bone by the screws establishes secure fixation. In locking screw-plate systems, the construct acts as a single beam in the bone, and the plate does not require compression and friction to achieve mechanical stability. Locking technology can potentially be advantageous when working with compromised bone as is the case with pathologic fractures or impending fractures, where bone quality may be less than ideal extending proximal and distal to the fracture or lesion. It is highly likely that with
Fig. 3 Failed internal fixation necessitating conversion to megaprosthesis. Anteroposterior left hip and proximal femur radiograph (a) shows a pathologic subtrochanteric fracture non-union with hardware failure of a reconstruction femoral nail in a previously irradiated proximal femur originally treated for metastatic breast carcinoma approximately 1 year earlier. Because of the previous irradiation and low likelihood for healing, the patient was treated with resection to below the fracture level and reconstruction with a long stem cemented megaprosthetic hip hemiarthroplasty (b). Pain relief was complete, and the patient was able to fully weight bear immediately after the procedure. At 1 year postoperatively, she usually walks without ambulatory aids but a Trendelenberg gait
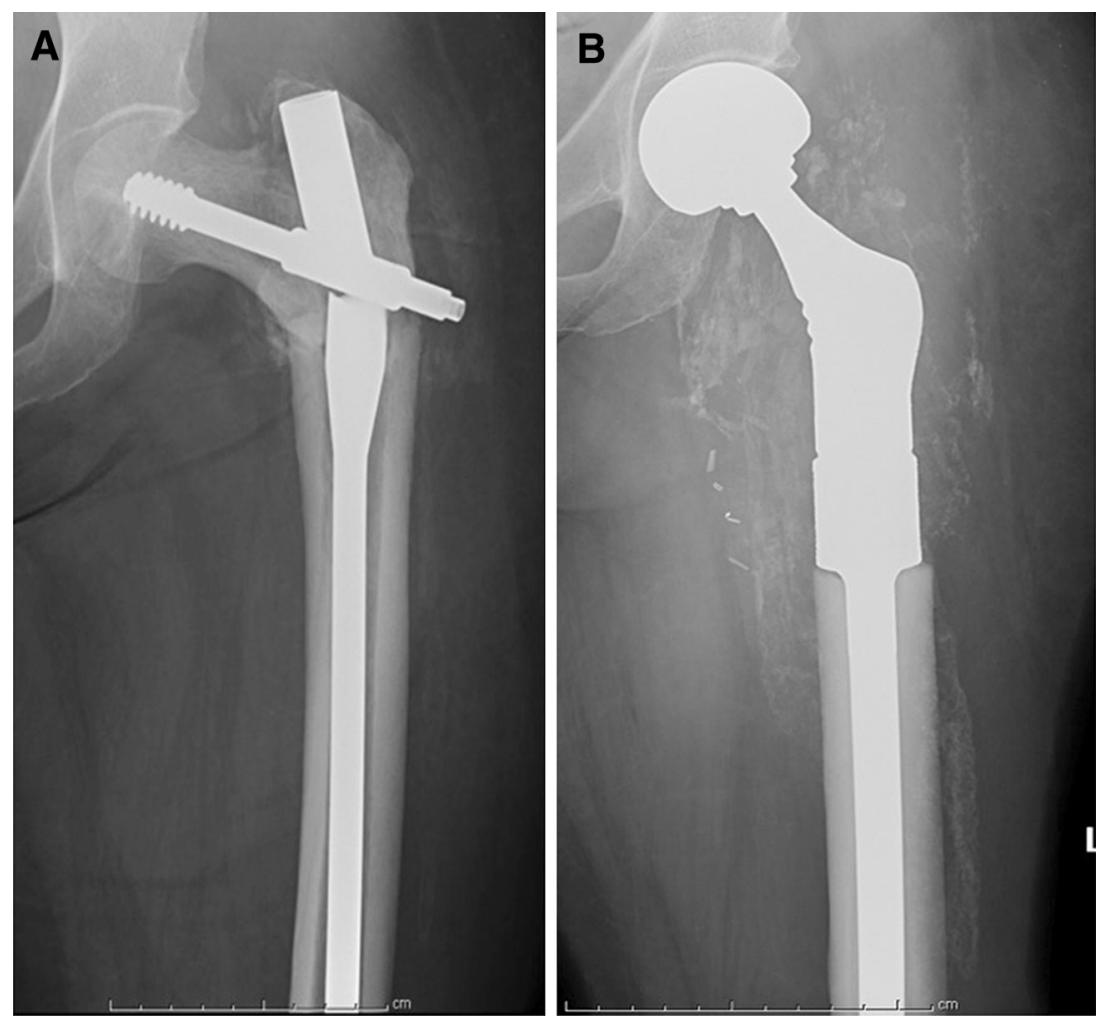
the increased availability and popularity of locked plating in the trauma community, surgeons treating pathologic fractures have implemented locking plates into their practice as well. However, reported results lag behind the clinical usage. The anatomic sites where locking plates would appear to have the most theoretical benefit are the distal femoral and proximal tibial peri-articular regions, but these are relatively uncommon sites of metastatic disease.

\section{Humeral Fracture Fixation}

Pathologic fractures and impending fractures of the humerus have been treated in a variety of ways, with much ongoing debate among surgeons. Although indications for operative intervention are generally accepted, the controversy lies in the specific methods of internal fixation. Treatments with intramedullary nails, plates, and arthroplasty have each been advocated in the literature [28-31].
Intramedullary nails are commonly used and offer several advantages over plate fixation. They can protect a longer segment of the bone (although not distal to the superior aspect of the olecranon fossa) compared to plates, the dissection needed is less than that for plate fixation, and rigid fixation can be achieved by combination with both interlocking screws and methylmethacrylate (although interlocking screw fixation alone is not generally considered truly rigid fixation). Plate fixation offers the advantage of avoiding damage or pain to the rotator cuff, better fixation in very proximal or distal fractures where adequate control of the fracture might not be possible with an intramedullary device, and more rigid fixation than can be achieved with intramedullary stabilization without bone cement. Arthroplasty is most useful when rigid fixation cannot be achieved with internal fixation or when the articular surfaces have been destroyed past the point of achieving adequate internal fixation. It offers the advantage
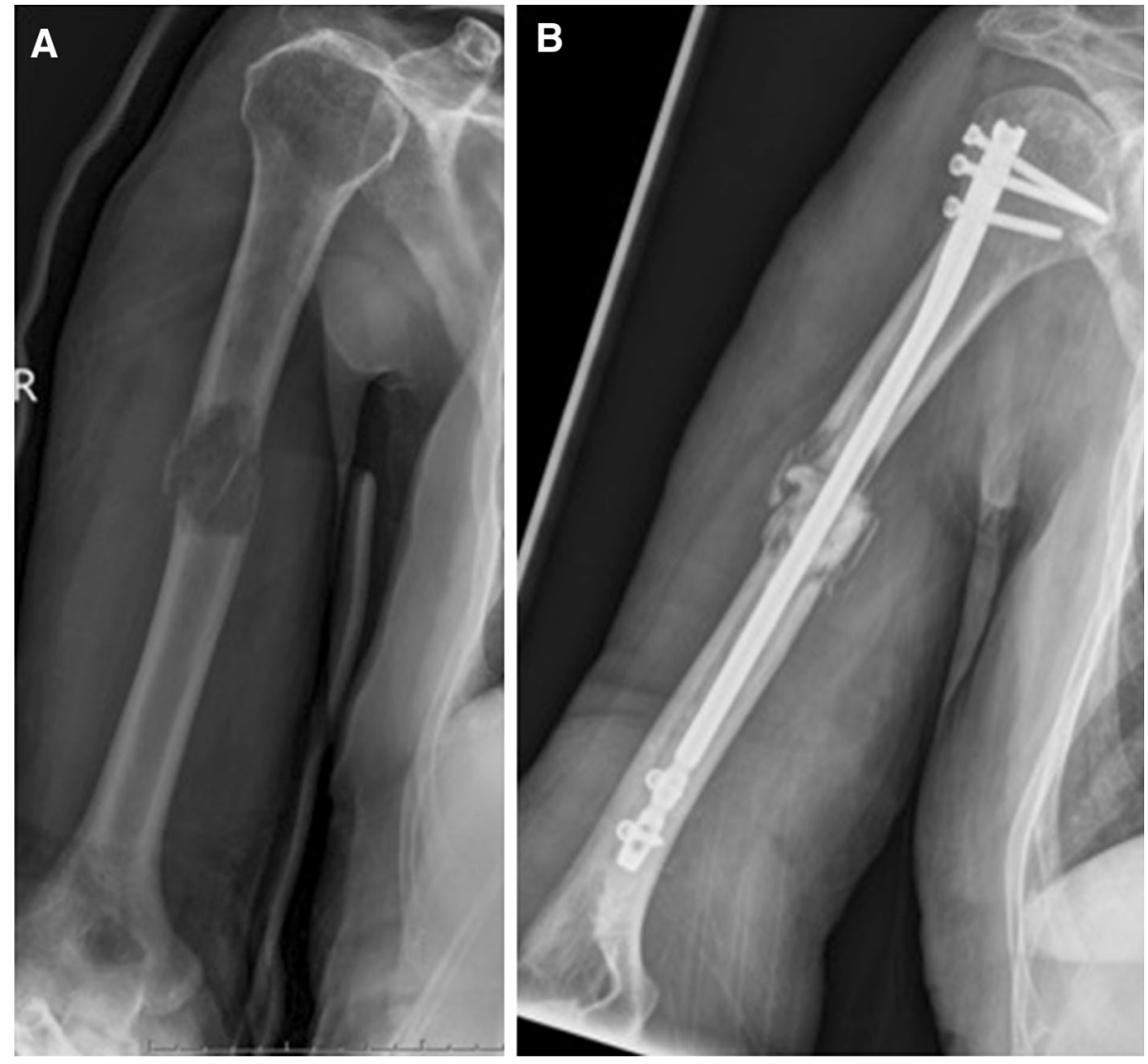

Fig. 4 Cemented humeral fixation. In this patient with a pathologic fracture of the midshaft of the humerus, the patient carried no preexisting diagnosis of cancer. Anteroposterior view of the right humerus shows a segmental defect at the level of the fracture (a). Hence, a biopsy and operative treatment of the humeral lesion were planned. Since the lesional site needed to be approached directly for the biopsy, after the frozen section confirmed the diagnosis of metastatic carcinoma, the lesion was thoroughly curetted, and an antegrade intramedullary humeral nail was cemented into the humerus using the technique described by Laitinen et al. (b) [24]. Other options for this lesion would have been a percutaneous needle biopsy followed by intramedullary humeral stabilization without cement, plating with cementing of the defect, and use of an intercalary segmental prosthetic defect device. This patient had complete early pain relief and restoration of shoulder and elbow function 
of not relying on stability of internal fixation devices, provides immediate pain relief and restoration of function of the more distal upper extremity (although shoulder function is poor), and is generally very durable. The latest literature for each of these treatment options will be reviewed in the following paragraphs.

Pathologic fractures and impending pathologic fractures of the humerus have been treated successfully with intramedullary nails [30, 32•]. Pretell et al. [30] reported good results on a series of 22 pathologic humeral shaft fractures treated with closed, unreamed, antegrade, locked, intramedullary nails. Seventeen of 19 patients reported that they were satisfied with their treatment. There were no complications related to the implants. This technique, which is relatively minimally invasive and does not require opening of the fracture or lesion site, has been the standard of care for diaphyseal lesions/fractures for some time. More recently, Laitinen et al. [32•] performed a retrospective review of 21 patients who underwent cemented intramedullary nailing for pathological fracture of the humeral shaft. In this series the tumor site was exposed, and, after curettage of the mass, cement was pressed into the canal both proximally at the insertion site and also proximally and distally from the fracture site to allow as much cement in the intramedullary canal as possible. These patients were compared to a historical control group of patients that underwent locked intramedullary nailing for pathological fractures. The patients in this single arm retrospective study treated with cemented intramedullary nailing had better pain relief, less use of analgesics, and better functional restoration immediately after the surgical procedure with no increase in surgical complications (Fig. 4).

Arthroplasty of the humerus for metastatic disease is a more invasive and often more technically demanding procedure than internal fixation, and it brings with it a host of potential complications unique from those of internal fixation, including subluxation/dislocation and loosening. Humeral arthroplasty is most commonly performed as a proximal humeral hemi-arthroplasty with a cemented longstem device, since most humeral metastatic disease is located in the proximal humerus. More extensive proximal destruction may require a proximal humeral megaprosthesis hemi-arthroplasty, but this sacrifices the rotator cuff insertion sites and does not reliably result in any meaningful restoration of shoulder function. For mid-diaphyseal humeral pathologic fractures with segmental bone destruction, intercalary spacer devices are available [3336]. However, because of concerns regarding early development of radiolucencies around the stems of these implants, their usage should be restricted to patients with limited life expectancy (Fig. 5). Recent reports strongly favor use of cemented stems over uncemented stems because of premature loosening with the latter.

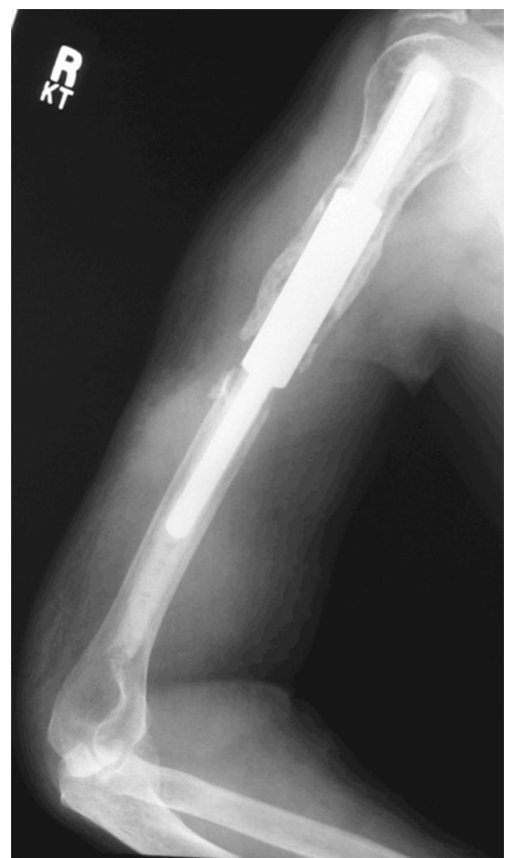

Fig. 5 Intercalary humeral spacers. Prosthetic reconstruction for humeral segmental defects has evolved to a lap joint construct with or without accompanying plate fixation, depending upon the manufacturer. In this case, an early generation intercalary humeral spacer was cemented proximally and distally. Note the healing bone surrounding the body portion of the implant but some evidence of radiolucency developing at the prosthetic-cement interface around the proximal stem

The distal humerus is the least likely area of the humerus to be involved by metastatic disease. Since intramedullary stabilization is not possible in this anatomic location, the options include 90:90 plating when adequate distal bone is available or prosthetic distal humeral reconstruction when bone is lacking. Funovics et al. [31] recently published a series where modular prosthetic reconstruction was used as the treatment for major bone defects of the distal humerus. The series included 53 elbows of which 38 were for tumor resection and 15 were for failed treatment of elbow joint degeneration. Overall complication rates were much lower for the tumor group than for the revision group being 24-60\%, respectively. Elbow function and clinical outcomes assessed with the Inglis-Pellicci scoring system were also better with the tumor group (85) compared to the revision group (77). The authors concluded that the modular prosthesis provides a stable reconstruction of the elbow with satisfactory function and disease control in patients with a tumor involving the distal humerus.

\section{Porous Tantalum Acetabular Reconstruction}

Metastatic processes commonly involve the bony pelvis. When this occurs in the periacetabular region, it can create 
substantial morbidity for patients with metastatic bone disease. Due to the short life expectancy after arthroplasty for patients with disseminated malignancy, 8.6 months in one series [37], functional outcomes are especially important for patients treated for metastatic disease of the acetabulum. Harrington developed specialized operative techniques to allow for adequate support and fixation of the acetabular component of a total hip prosthesis [3, 38]. These techniques were divided into subgroups based on the specific biomechanical deficiencies in the periacetabular bone. The goal of each was to support a total hip prosthesis in its normal anatomical position and to allow transmission of weight-bearing forces into bone.
Marco et al. [39] in 2000 reported on their series of 55 patients with metastatic disease of the acetabulum that were treated with operative acetabular reconstruction combined with a total hip arthroplasty. Fifty-four of the hips were reconstructed with a protrusio cup and one patient was treated with a hemi-pelvis endoprosthesis. Large defects were reinforced with a cement and pin or screw fixation (the modified Harrington technique). Thirtyfour patients $(76 \%)$ had relief of pain. Nine of the 18 patients who could not walk preoperatively regained the ability to ambulate after surgery. Fourteen of the 17 patients who were community ambulators retained that ability after surgery.
Fig. 6 Tantalum cup reconstruction. This patient with known metastatic endometrial cancer involving the left periacetabular region had severe pain despite irradiation to the hemi-pelvis. Anteroposterior left hip radiograph shows acetabular destruction and protrusio deformity (a). Coronal CT scan shows the extent of the central and cephalad defect but an intact peripheral rim (b). The patient underwent placement of a tantalum cup articulating with the intact but weakened acetabular rim over a cemented defect (c). In addition, a cage was then placed over the cup to further distribute the forces to the intact bone in the more cephalad and caudad bone and secured with numerous screws placed in the bone and cement. One year postoperatively, the patient ambulates with a walker outside the home
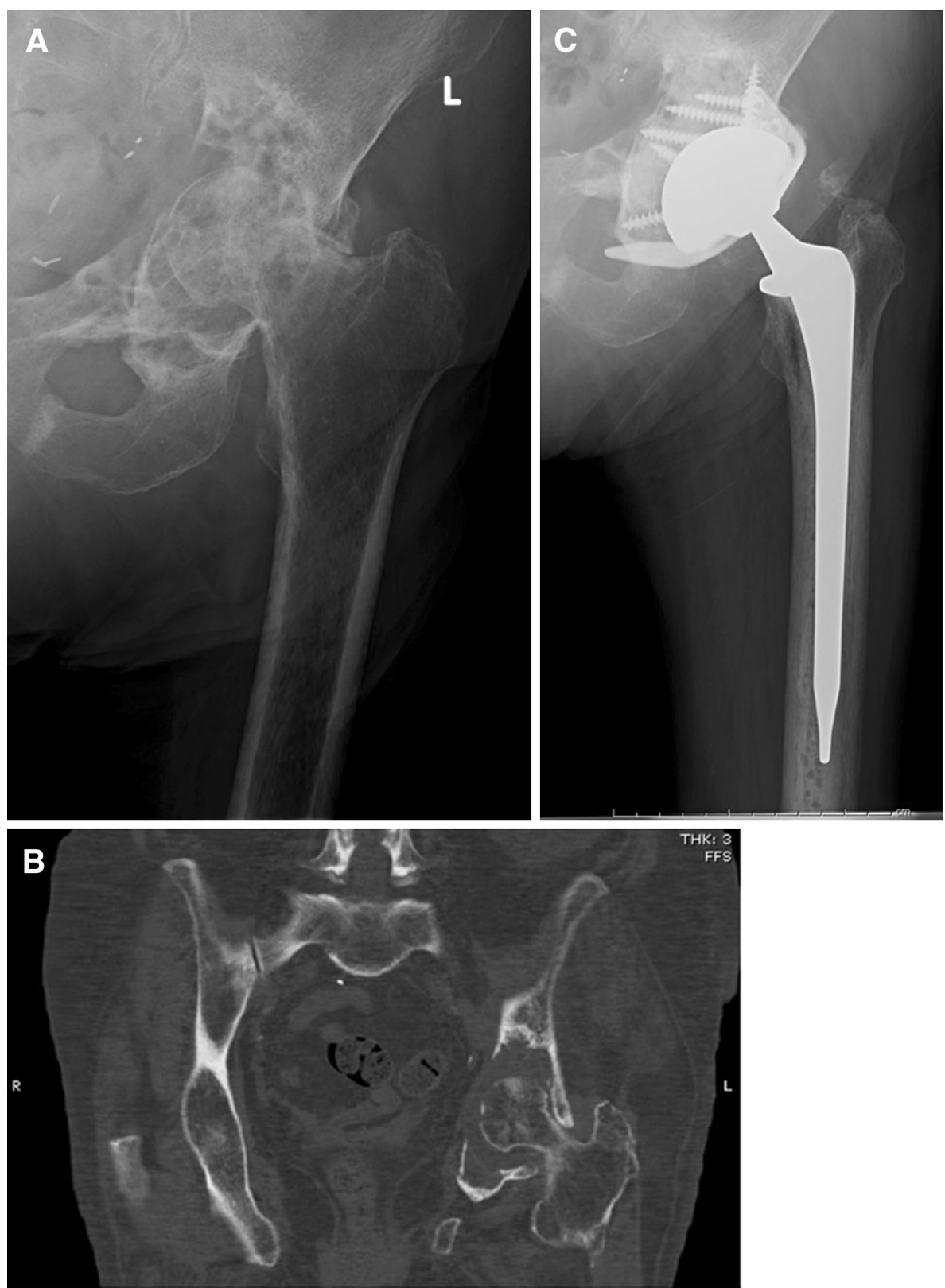
Pelvic irradiation has been long recognized as an independent adverse factor in the outcome of total hip arthroplasty, even in patients without active pelvic metastatic disease [40]. One series reported a $52 \%$ rate of acetabular component loosening using cemented components at mean 69-month follow-up [40]. Their results only improved to $19 \%$ acetabular aseptic loosening at mean 40 months follow-up with use of reinforcement rings. Because of these difficulties, tantalum acetabular components and augments have begun to be used for total hip arthroplasty in this setting [41, 42]. Porous tantalum acetabular implants have potential benefits for acetabular component fixation in challenging situations such as a history of or need for pelvic irradiation. The material has a high coefficient of friction, potential for ingrowth, and encouraging results when used for primary and revision total hip arthroplasty in traditional arthroplasty patient populations.

Rose et al. reported preliminary results for 11 patients with 12 total hip arthroplasties who had therapeutic pelvic radiation to treat underlying metastatic bone disease prior to arthroplasty [41]. No clinical failures occurred at a mean of 31 month follow-up and no reoperations. Harris hip scores improved from an average of 46 points to 88 points postoperatively. More recently, in 2012, Khan et al. [42••] reported the longer term follow-up (mean 56 months) for a series of 20 consecutive patients from the same institution with large destructive nonprimary periacetabular tumors, of which 15 had received preoperative radiation. In this series a large uncemented porous tantalum acetabular shell (sometimes with tantalum augments) was fixed to the remaining bone after local tumor curettage. The Harris hip scores improved from a mean of 32 preoperatively to a mean of 74 postoperatively. There were no cases of progressive radiolucent lines or component migration.

Hence, it would appear that the use of tantalum acetabular component reconstruction represents a major advance over standard cemented acetabular components and protrusio cages in the surgical treatment of peri-acetabular metastatic disease requiring irradiation. These devices may be used alone or in combination with augments or "cage-in-cup" constructs (Fig. 6).

\section{Cementoplasty (Osteoplasty)}

With the push for more minimally invasive surgery and on the heels of vertebroplasty for spinal metastatic disease, cementoplasty (osteoplasty) has developed as a means to address painful pelvic metastases [43-49]. It generally involves the percutaneous palliative debulking of pelvic metastases and filling with methylmethacrylate bone cement, and it may be done alone or in combination with ablative treatments such as radiofrequency ablation (RFA) and cryotherapy (see below).
The procedure is done under CT or fluoroscopy guidance. The earliest report in 2000 described multi-institutional retrospective findings in 18 patients with pelvic or sacral bone metastases [47]. Overall, $82 \%$ of patients achieved improvement in pain and walking, but one patient progressed to acetabular fracture 2 weeks postoperatively. Other reports have lumped the results of vertebroplasty and acetabuloplasty. Most recently, in a series of 92 patients with lung cancer metastases, visual analog scale, and Karnofsky Performance Scale scores improved significantly [48]. Among the 22 acetabuloplasty patients in that series, Harris hip scores improved significantly. No major complications were reported in either that series or in another combining osteoplasty with RFA in 28 lesions of the thoracic and lumbar spine, sacrum, pelvis, acetabulum, femur, and tibia [49].

\section{Minimally and Non-Invasive Treatment Advancements}

For patients with metastatic disease who either do not require surgical treatment or who are poor candidates for surgery, alternative treatments are employed. Although external beam radiation therapy has been the standard of care in this situation, 20-30\% of patients treated with this modality do not experience pain relief [50]. As a complement to radiotherapy, several image-guided ablation strategies are now available for pain associated with bone metastases, including RFA, cryoablation, image-guided focused ultrasound, ethanol, and laser-induced interstitial thermotherapy. In these situations minimally invasive procedures and advancements in this area have become increasingly more popular for the treatment of bone metastases. These image-guided percutaneous methods have proven to be effective and safe over the past decade. The following discussion focuses on RFA, cryoablation, and image-guided focused ultrasound.

\section{Radiofrequency Ablation}

Radiofrequency ablation (RFA), traditionally used to treat tumors of the liver and kidney, cardiac arrhythmias, unresectable pulmonary tumors, facet osteoarthritis, and osteoid osteomas, has more recently been used in the treatment of painful metastatic bone disease [49-52].

Callstrom et al. [50] reported on RFA in a series of patients who had persistent pain after failed radiation therapy or chemotherapy. Significant reduction in pain by visual analog pain scores was observed following the procedures and measured through 4 weeks postoperatively; eight out of ten patients using analgesics reported reduced use, and no serious complications were observed. Goetz et al. [53] reported similar good RFA results in 43 patients using a similar study design but expanded to a multicenter study at longer follow-up. The mean worst pain was 7.9 prior to the procedure and decreased to $4.5,3.0$, and 1.4 at 
4, 12, and 24 weeks following RFA, respectively. Fortyone of 43 patients experienced a decrease in pain that was considered clinically significant, opioid usage significantly decreased at weeks 8 and 12, and only three adverse events were reported. A second multicenter RFA trial was published in 2010 by Dupuy et al. [54]. In this series, 55 patients with intractable pain due to bone metastases not being treated concomitantly with external beam radiation or chemotherapy received RFA. RFA reduced pain by all pain assessment measures and improved mood at 1 and 3 months, and it resulted in grade 3 toxicities in only $5 \%$ (3) of patients.

RFA is a well-studied, minimally invasive ablative technique that appears to be effective and safe for treatment for pain secondary to bony metastatic disease. Early results have been reported in combination with cementoplasty, as previously described [49]. Of the several different image-guided ablation strategies, RFA remains the most widely used. Significant pain reduction is possible even in those patients who have failed treatment from external beam radiation therapy and chemotherapy.

\section{Cryoablation}

Cryoablation is another method of image-guided minimally invasive treatment for the palliation of painful metastatic tumors involving the bone. Not unlike RFA, cryoablation was initially used for non-osseous lesions such as hepatic and renal tumors $[55,56]$. Cryoablation has become a more effective method of treatment as technology has advanced. The use of freezing temperatures for therapeutic destruction of tissue was first described in England in 1845 with the use of iced salt solutions to freeze certain cancerous tumors. This resulted in a reduction of tumor size and pain $[57,58]$. Improved freezing techniques were possible with the development of solidified carbon dioxide, liquid nitrogen, and nitrous oxide. This technique has become more feasible at other disease sites with the development of an argon-based system, smaller applicator diameters, sealed cryoprobes, and improved insulation.

Callstrom et al. [59] initially reported on the results of 14 patients treated with cryoablation for one or two painful metastatic bone lesions. Patient pain was measured using the Brief Pain Inventory prior to the procedure and measured at scheduled intervals up to 6 months. Mean pretreatment score for worst pain in a 24-h period was 6.7 of 10; this decreased to 3.84 weeks after treatment. Mean pain interference with activities of daily living was 5.5 of 10 before treatment and decreased to 3.24 weeks after treatment. All patients for whom narcotics were prescribed prior to the procedure reported a reduction in these medications after cryoablation. No serious complications were observed during this series.
More recently, the same group reported results in a follow-up, multicenter trial on 61 patients treated with cryoablation for the same indications [60••]. The mean score for worst pain in a 24 -h period was 7.1 of 10 prior to cryoablation and decreased to 5.1, 4.0, 3.6, and 1.4 at 1,4 , 8 , and 24 weeks, respectively, with all results being statistically significant. One patient had a major complication resulting in osteomyelitis at the site of ablation.

Advocates of cryoablation suggest that it is advantageous over RFA for two reasons. First, the ablation zone of cryoablation is readily identifiable with $\mathrm{CT}$ imaging as a low-attenuation ice ball beyond which tissues are safe from thermal injury. RFA's critical limitation is the non-visualization of the ablation margin with computed tomography monitoring. Second, cryoablation appears not to increase pain during the procedure or in the immediate posttreatment period, which RFA has been reported to do. Although not studied as thoroughly as RFA for metastatic bone disease, recent publications indicate that it is a safe and effective method of treating metastatic bone disease.

\section{High-Intensity Focused Ultrasound}

High-intensity focused ultrasound (HIFU) ablation is a completely noninvasive method for the treatment of localized tumors [61]. An ultrasound beam is focused onto the tumor as it passes through the tissue. This enables the use of an external ultrasound energy source to induce thermal ablation of a tumor at a depth under the intact skin. Under the guidance of real-time ultrasonographic imaging and/or magnetic resonance imaging, the motion of a therapeutic transducer can facilitate ablation of a threedimensional target. The temperature at the targeted position increases to approximately $65-100{ }^{\circ} \mathrm{C}$ in $0.5-1.0 \mathrm{~s}$. The tumor tissues undergo coagulative necrosis with little damage to nearby healthy tissues.

Li et al. [62] reported results on the use of HIFU on 25 patients with malignant bone tumors. Of the 25 patients, 13 had a primary bone tumor, and 12 had metastatic bone disease. After HIFU, $100 \%$ of patients experienced significant pain relief with $87.5 \%$ of patients experiencing complete pain relief. Of the 12 patients with metastatic disease, 5 had complete response, 4 had partial response, 1 had moderate response, 1 had stable disease, and 1 had progressive disease. Hence, although further studies are needed, the early reports on the use of HIFU in this setting appear promising.

\section{Conclusions}

Treatments for metastatic bone disease are developing and evolving with advancements in technology and surgical techniques. These developments will hopefully continue to 
improve our care for patients with metastatic bone disease, keeping in mind the basic principles of relieving pain, gaining local tumor control, and maintaining or regaining functional status.

\section{Compliance with Ethics Guidelines}

Conflict of Interest Timothy A. Damron has received research grant support from Stryker, Cempra, Zetrox, Wright Medical, the National Institutes of Health, the National Cancer Institute, the Carol Baldwin Breast Cancer Foundation, and the Musculoskeletal Tumor Society; has received royalties form Lippincott Williams \& Wilkins; and has had travel/accommodations expenses covered/reimbursed by Stryker. Brian J. Kistler declares that he has no conflict of interest.

Human and Animal Rights and Informed Consent This article does not contain any studies with animal subjects performed by any of the authors. With regard to the authors' research cited in this paper, all procedures were followed in accordance with the ethical standards of the responsible committee on human experimentation and with the Helsinki Declaration of 1975, as revised in 2000 and 2008.

\section{References}

Recently published papers of particular interest have been highlighted as:

- Of importance

-. Of major importance

1. Schulman KL, Kohles J. Economic burden of metastatic bone disease in the US. Cancer. 2007;109:2334-42.

2. Harrington KD. Orthopedic surgical management of skeletal complications of malignancy. Cancer. 2000;80:1614-27.

3. Bickels J, Dadia S, Lidar Z. Surgical management of metastatic bone disease. J Bone Joint Surg Am. 2009;91:1503-16.

4. Biermann JS, Holt GE, Lewis VO, Schwartz HS, Yaszemski MJ. Metastatic bone disease: diagnosis, evaluation, and treatment. J Bone Joint Surg Am. 2009;91:1518-30.

5. Varadhachary GR, Abbruzzese JL, Lenzi R. Diagnostic strategies for unknown primary cancer. Cancer. 2004;100:1776-85.

6. Jacofsky DJ, Papagelopoulos PJ, Sim FH. Advances and challenges in the surgical treatment of metastatic bone disease. Clin Orthop Relat Res. 2003;415S:S14-8.

7. Ward WG, Holsenbeck S, Dorey FJ, Spang J, Howe D. Metastatic disease of the femur: surgical treatment. Clin Orthop Relat Res. 2003;415:S230-44

8. Mirels H. Metastatic disease in long bones. A proposed scoring system for diagnosing impending pathologic fractures. Clin Orthop Relat Res. 1989;249:256-64.

9. Damron TA, Morgan H, Prakash D, Grant W, Aronowitz J, Heiner J. Critical evaluation of Mirels' rating system for impending pathologic fractures. Clin Orthop Relat Res. 2003;415:S201-7.

10. Damron TA, Ward WG. Risk of pathologic fracture: assessment. Clin Orthop Relat Res. 2003;415:S208-11.

11. Evans AR, Bottro J, Grant W, Chen BY, Damron TA. Mirel's rating for humerus lesions is both reproducible and valid. Clin Orthop Relat Res. 2008;466:1279-84.

12. El-Husseiny $M$, Coleman N. Inter- and intra-observer variation in classification systems for impending fractures of bone metastases. Skeletal Radiol. 2010;39:155-60.
13. Van der linden YM, Dijkstra PD, Kroon HM, Lok JJ, Noordijk EM, Leer JW, Marijnen CA. Comparative analysis of risk factors for pathological fracture with femoral metastases. J Bone Joint Surg-Br. 2004;86(4):566-73.

14. Snyder BD, Hauser-Kara DA, Hipp JA, Zurakowski D, Hecht AC, Gebhardt MC. Predicting fracture through benign skeletal lesions with quantitative computed tomography. J Bone Joint Surg Am. 2006;88:55-70.

15. Leong NL, Anderson ME, Gebhardt M, Snyder BD. Computed tomography-based structural analysis for predicting fracture risk in children with benign skeletal neoplasms. Comparison of specificity with that of plain radiographs. J Bone Joint Surg Am. 2010;92:1827-33.

16. Anez-Bustillos L, Derikx LC, Verdonschot N, Calderon N, Zurakowski D, Snyder BD, Nazarian A, Tanck E. Finite element analysis and CT-based structural rigidity analysis to assess failure load in bones with simulated lytic defects. Bone. 2013;58:160-7. doi:10.1016/j.bone.2013.10.009.

17. Mann KA, Lee J, Arrington SA, Damron TA, Allen MJ. Predicting distal femur bone strength in a murine model of tumor osteolysis. Clin Orthop Relat Res. 2008;466(6):1271-8.

18. Alvi HM, Damron TA. Prophylactic stabilization for bone metastases, myeloma, or lymphoma: do we need to protect the entire bone? Clin Orthop Relat Res. 2013;471(3):706-14.

19. Herrenbruck T, Erickson EW, Damron TA, Heiner J. Adverse clinical events during cemented long-stem femoral arthroplasty. Clin Orthop Relat Res. 2002;395:154-63.

20. Frassica FJ, Frassica DA. Evaluation and treatment of metastases to the humerus. Clin Orthop Relat Res. 2003;415S:S212-8.

21. Sarahrudi K, Greitbauer M, Platzer P, et al. Surgical treatment of metastatic fractures of the femur: a retrospective analysis of 142 patients. J Trauma. 2009;66:1158.

22. Steensma M, Boland PJ, Morris CD, et al. Endoprosthetic treatment is more durable for pathologic proximal femur fractures. Clin Orthop Relat Res. 2012;470:920.

23. - Harvey N, Ahlmann ER, Allison DC, et al. Endoprostheses last longer than intramedullary devices in proximal femur metastases. Clin Orthop Relat Res 2012; 470:684. Recent article suggesting shift to arthroplasty is favorable to internal fixation for pathologic proximal femur metastases.

24. Frigg R. Development of the locking compression plate. Injury. 2003;34:SB6-10.

25. Ricci W, Haidukewych G. Locked plating in orthopaedic trauma: a clinical update. J Am Acad Orthop Surg. 2008;16:347-55.

26. Gardner MJ, Griffith MH, Demetrakopoulos D, et al. Hybrid locked plating of osteoporotic fractures of the humerus. J Bone Joint Surg Am. 2006;88:1962-7.

27. Tejwani NC, Guerado E. Improving fixation of the osteoporotic fracture: the role of locked plating. J Orthop Trauma. 2011;25:S56-60.

28. Damron TA, Heiner JP, Freund EM, et al. A biomechanical analysis of prophylactic fixation for pathologic fractures of the distal third of the humerus. J Bone Joint Surg Am. 1994;76A:839-47.

29. Damron TA, Rock MG, Choudhury SN, et al. Biomechanical analysis of prophylactic fixation for middle third humeral impending pathologic fractures. Clin Orthop Relat Res. 1999;363:240-8.

30. Pretell J, Rodriguez J, Blanco D, Zafra A, Resines C. Treatment of pathological humeral shaft fractures with intramedullary nailing. A retrospective study. Int Orthop. 2010;34:559-63.

31. Funovics PT, Schuh R, Adams SB, Sabeti-Aschraf M, Dominkus M, Kotz RI. Modular prosthetic reconstruction of major bone defects of the distal end of the humerus. J Bone Joint Surg Am. 2011;93:1064-74.

32. - Laitinen M, Nieminen J, Pakarinen T. Treatment of pathological humerus shaft fractures with intramedullary nails with or without cement fixation. Arch Orthop Trauma Surg. 2011;131:503-8 Treatment with cement fixation superior to uncemented humeral nails. 
33. Damron TA, Leerapun T, Hugate RR, Shives TC, Sim FH. Does the second-generation intercalary humeral spacer improve on the first? Clin Orthop Relat Res. 2008;466(6):1309-17.

34. Henry JC, Damron TA, Weiner MM, Higgins ME, Werner FW, Sim FH. Biomechanical analysis of humeral diaphyseal segmental defect fixation. Clin Orthop Relat Res. 2002;396:231-9.

35. Damron TA, Sim FH, Shives TC, An KN, Rock MG, Pritchard DJ. Intercalary spacers in the treatment of segmentally destructive diaphyseal humeral lesions in disseminated malignancies. Clin Orthop Relat Res. 1996;324:233-43.

36. Ahlmann ER, Menendez LR. Intercalary endoprosthetic reconstruction for diaphyseal bone tumours. J Bone Joint Surg Br. 2006;88(11):1487-91.

37. Schneiderbauer MM, Von Knoch M, Schleck CD, Harmsen WS, Sim FH, Scully SP. Patient survival after hip arthroplasty for metastatic disease of the hip. J Bone Joint Surg Am. 2004;86A:1684-9.

38. Harrington KD. The management of acetabular insufficiency secondary to metastatic malignant disease. J Bone Joint Surg Am. 1981;63:653-64.

39. Marco RA, Sheth DS, Boland PJ, Wunder JS, Siegel JA, Healey JH. Functional and oncological outcome of acetabular reconstruction for the treatment of metastatic disease. J Bone Joint Surg Am. 2000;82:642-51.

40. Massin P, Duparc J. Total hip replacement in irradiated hips. J Bone Joint Surg Br. 1995;77:847-52.

41. Rose PS, Halasy M, Trousdale RT, Hanssen AD, Sim FH, Berry DJ, Lewallen DG. Preliminary results of tantalum acetabular components for THA after pelvic radiation. Clin Orthop Relat Res. 2006;453:195-8.

42. • Khan FA, Rose PS, Yanagisawa M, Lewallen DG, Sims FH. Porous Tantalum Reconstruction for Destructive Nonprimary Periacetabular Tumors. Clin Orthop Relat Res. 2012;470:594-601 Successful treatment of large pathologic bone defects of the pelvis treated with tantalum acetabular shell.

43. Deschamps F, de Baere T. Cementoplasty of bone metastases. Diagn Interv Imaging. 2012;93(9):685-9.

44. Maccauro G, Liuzza F, Scaramuzzo L, et al. Percutaneous acetabuloplaty for metastatic acetabular lesions. BMC Musculoskelet Disord. 2008;9:66.

45. Cotton A, Deprez X, Migaud H, Chabanne B, Duquesnoy B, Chastanet P. Malignant acetabular osteolyses: percutaneous injection of acrylic bone cement. Radiology. 1995;197(1):307-10.

46. Scaramuzzo L, Maccauro G, Rossi B, Messuti L, Maffulli N, Logroscino CA. Quality of life in patients following percutaneous PMMA acetabuloplasty for acetabular metastases due to carcinoma. Acta Orthop Belg. 2009;75(4):484-9.

47. Marcy PY, Palussière J, Descamps B, Magné N, Bondiau PY, Ciais C, Bruneton JN. Percutaneous cementoplasty for pelvic bone metastasis. Support Care Cancer. 2000;8(6):500-3.

48. Wang Z, Zhen Y, Wu C, Li H, Yang Y, Shen Z, Zhao H, Yao Y. CT fluoroscopy-guided percutaneous osteoplasty for the treatment of osteolytic lung cancer bone metastases to the spine and pelvis. J Vasc Interv Radiol. 2012;23(9):1135-42.

49. Hoffmann RT, Jakobs TF, Trumm C, Weber C, Helmberger TK, Reiser MF. Radiofrequency ablation in combination with osteoplasty in the treatment of painful metastatic bone disease. J Vasc Interv Radiol. 2008;19(3):419-25.

50. Callstrom MR, Charboneau JW, Goetz MP, Rubin J, Atwell TD, Farrell MA, Welch TJ, Maus TP. Image-guided ablation of painful metastatic bone tumors: a new and effective approach to a difficult problem. Skeletal Radiol. 2006;35:1-15.

51. Dupuy DE, Hong R, Oliver B, Goldberg SN. Radiofrequency ablation of spinal tumors: temperature distribution in the spinal canal. AJR Am J Roentgenol. 2000;175:1263-6.

52. Callstrom MR, Charboneau JW, Goetz MP, Rubin J, Wong GY, Sloan JA, Novotny PJ, Lewis BD, Welch TJ, Farrell MA, Maus TP, Lee RA, Reading CC, Petersen IA, Pickett DD. Painful metastases involving bone: feasibility of percutaneous CT- and US-guided radio-frequency ablation. Radiology. 2002;224:87-97.

53. Goetz MP, Callstrom MR, Charboneau JW, Farrell MA, Maus TP, Welch TJ, Wong GY, Sloan JA, Novotny PJ, Petersen IA, Beres RA, Regge D, Capanna R, Saker MB, Gronemeyer DH, Gevargez A, Ahrar K, Choti MA, de Baere TJ, Rubin J. Percutaneous image guided radiofrequency ablation of painful metastases involving bone: a multicenter study. J Clin Oncol. 2004;22:300-6.

54. Dupuy DE, Liu D, Hartfeil D, et al. Percutaneous radiofrequency ablation of painful osseous metastases: a multicenter American College of Radiology Imaging Network trial. Cancer. 2010;116:989-97.

55. Sewell PE, Howard JC, Shingleton WB, Harrison RB. Interventional magnetic resonance image-guided percutaneous cryoablation of renal tumors. South Med J. 2003;96:708-10.

56. Callstrom MR, Charboneau JW. Technologies for ablation of hepatocellular carcinoma. Gastroenterology. 2008;134:1831-5.

57. Gage AA. History of cryosurgery. Semin Surg Oncol. 1998;14: 99-109.

58. Gage AA, Gonder MJ, Soanes WA, Emmings FG. Cancer cryotherapy. Mil Med. 1967;132:550-6.

59. Callstrom MR, Atwell TD, Charboneau JW, et al. Painful metastases involving bone: percutaneous image-guided cryoablationprospective trial interim analysis. Radiology. 2006;241:572-80.

60. • Callstrom MR, Dupuy DE, Solomon SB, et al. Percutaneous Image-Guided Cryoablation of Painful Metastases Involving Bone, Multicenter Trial. Cancer. 2013;119:1033-41 Multicenter trial showing significant decrease in pain following cryoablation.

61. Chuanxing L, Weidong Z, Weijun F, Jinhua H, Fujun Z, Peihong W. Noninvasive treatment of malignant bone tumors using highintensity focused ultrasound. Cancer. 2010;116:3934-42.

62. Li C, Zhang W, Fan W, Huang J, Zhang F, Wu P. Noninvasive treatment of malignant bone tumors using high-intensity focused ultrasound. Cancer. 2010;116(16):3934-42. 